\title{
Stochastic climate models, part 3. Application to zonally averaged energy models
}

\author{
By P. LEMKE, Max-Planck-Institut für Meteorologie, \\ Bundesstrasse 55, D-2000 Hamburg 13, BRD
}

(Manuscript received December 10, 1976; in final form February 4, 1977)

\begin{abstract}
A stochastic Budyko-Sellers model is considered in which, in contrast to the usual statistical dynamical climate models, the nonaveraged weather fluctuations are retained as internal random forcing terms. Consequently, the climate variables are no longer deterministic but are stochastic variables, which can be characterized by their variance spectra. The calculated variance spectra of the yearly and zonally averaged surface temperature of the earth are consistent with observations both in the qualitative structure of the spectrum and the order of magnitude of the energy levels.
\end{abstract}

\section{Introduction}

In Part 1 of this series (Hasselmann, 1976, referred to in the following as I) a stochastic model of climate variability was considered in which slow changes of climate were explained as the integral response to continuous internal random forcing by short time-scale "weather" disturbances.

In Part 2 (Frankignoul \& Hasselmann, 1977) the concepts of I were applied to sea-surface temperature anomalies and thermocline variability in a local ocean-response model, which was valid for short-time scales of the order of 1 month to a few years.

In this paper a non-local and global stochastic climate model applicable for longer time scales is considered. Attention is again directed more toward the principal features of stochastic climate models than the quantitative modelling of climate variability. Thus the simplest climate model of Budyko (1969) and Sellers (1969) is used rather than later, more sophisticated models of Sellers (1973), Adem (1965), or others.

Several attempts have been made to explain climatic changes using statistical dynamical models (SDMs) in terms of changes in external parameters such as the solar radiation. The simplest of these models are the zonally averaged energy balance models of the Budyko-Sellers type. The characteristic feature of these models is the temperature dependent albedo, which provides a strong positive feedback between ice and snow cover and temperature. As a consequence of this positive feedback Budyko (1969) and Sellers (1969) found a great sensitivity of the equilibrium climate state to rather small changes in the solar radiative heating.

To close the prognostic climate equations of the SDM model, the short time-scale "weather terms", i.e. the meridional atmospheric fluxes of sensible and latent heat, are averaged and parameterized in terms of the surface temperature, while neglecting their fluctuations. In this way time variability can arise only through changes in the external parameters. However, if the statistical dynamical model is extended to retain also the non-averaged weather components, there is no need to invoke changes in external parameters to explain climate variability.

Because the weather fluctuations formally appear as random forcing terms in the prognostic climate equation, the climate variables are no longer deterministic, but have to be regarded also as stochastic variables and must accordingly be characterized by their power spectra (I) or similar statistical functions.

In this paper the power spectra of the surface 
temperature at different latitudes are calculated from a stochastic Budyko-Sellers model in which the ocean, the dominant contribution to the thermal inertia of the system, is represented by either a single layer or a two-layer model (Sections $2 a, 2 b$, respectively).

The parameterization of the mean weather terms, i.e. albedo, infrared emission and meridional flux, is described in Section 2c. For the temperature dependence of the albedo two parametrizations are used, Sellers' (1969) original expression and a modified formula. The power spectrum of the random forcing, providing the input to the model, is not available directly from meteorological data, but can be estimated with the aid of scaling considerations (Section 2d). The results of the model presented in Section 3 shows reasonable qualitative and order of magnitude agreement with observed climatic variance spectra.

\section{The stochastic model}

The basic climate variable in Budyko's (1969) and Sellers' (1969) models is the zonally and yearly averaged surface temperature $T$ of the earth. It is determined from the zonally averaged, vertically integrated equation of the heat balance of the earthatmosphere system,

$\int c_{p} \rho \frac{\partial T(r, \theta, \varphi, t)}{\partial t} d r=R(T)+A(T)$

where $R$ is the radiation balance

$R(T)=Q(1-\alpha(T))-I(T)$

and $A$ is the energy gain due to the meridional flux

$A=\frac{1}{a \cos \theta} \cdot \frac{\partial}{\partial \theta}(F \cos \theta)$

Here $c_{p}$ and $\rho$ represent the specific heat at constant pressure and the density respectively, $Q(\theta)$ is the mean annual incoming solar radiation per unit area at latitude $\theta ; \alpha(T)$ is the planetary albedo, $I(T)$ the infrared emission, $F$ the vertically integrated meridional heat flux, and $a$ the average radius of the earth.

The vertical integration in (2.1) extends from the upper atmosphere down to a depth of the ocean or land mass where the thermal response to meteorological forcing becomes negligible. The heat capacities of the atmosphere and the upper layers of the land masses are very small compared to that of the oceans, so that the vertical integration in (2.1) can be restricted to an integration over the effective thermal depth $h$ of the ocean (to be defined below). The left-hand side of (2.1) then has to be weighted with the factor $l(\theta)=l_{w}(\theta) / l_{0}(\theta)$, where $l_{0}$ is the length of a latitudinal circle and $l_{w}$ represents the length of the ocean-covered portion of $l_{0}$.

In this paper the earth is taken to be symmetric about the equator. Taking a spacing of $5^{\circ}$ latitude eq. (2.1) will be discretized in our numerical investigations into the following set of differential equations $(i=1, \ldots, 19)$

$c \rho l_{(i)} \frac{\partial}{\partial t} \int_{a-h}^{a} T_{i}(r, t) d r=R_{i}(T)+A_{i}(T)$

Equation (2.4) is usually used to test the sensitivity of the equilibrium climate state to changes in the solar radiation $Q$ (Schneider \& Gal-Chen, 1973; Held \& Suarez, 1974). To close the problem the right-hand side of (2.4) is averaged over a period sufficiently long to remove the weather fluctuations, and the "mean weather" is then parameterized in terms of the surface temperature.

Weather-fluctuation terms occur not only through the meridional heat flux, but also in the albedo and infrared emission terms through the variable cloudiness and humidity. In general, the righthand side of (2.4) can therefore be written as a mean plus the deviation from the mean

$R_{i}+A_{i}=\left\langle R_{i}\right\rangle+\left\langle A_{i}\right\rangle+S_{i}^{\prime}$

where $S_{i}^{\prime}=R_{i}^{\prime}+A_{i}^{\prime}$. In contrast to the usual SDM approach, we shall retain the term $S_{i}^{\prime}$ in the basic climate equation (2.4) as a stochastic forcing term. Thus the surface temperature is not determined deterministically (by (2.4)), but must be regarded as a stochastic variable.

\section{(a) One layer ocean model}

In applications of the Budyko-Sellers model to study non-stationary phenomena (Held \& Suarez, 1974; Schneider \& Gal-Chen, 1973) the ocean is normally described by a completely mixed homogeneous layer of depth $h_{1}$. Equation (2.4) then reduces to

$c \rho l_{(i)} h_{1} \frac{\partial T_{i}(a, t)}{\partial t}=\left\langle R_{i}\left(T_{i}(a, t)\right)\right\rangle+\left\langle A_{i}(T(a, t))\right\rangle+S_{i}^{\prime}$ 
For small excursions about an equilibrium state $T_{i}(a, t)$ (linear feedback) the quantities $\left\langle R_{l}\right\rangle$ and $\left\langle A_{i}\right\rangle$ can be expanded with respect to the surface temperature, and after Fourier transformation the basic climate equation may be written

$$
\begin{gathered}
i \omega T_{i}(a, \omega)=V_{i j}\left[T_{j}(a, \omega)-\hat{T}_{j}(a, \omega)\right]+ \\
\frac{1}{c \rho l_{(i)} h_{1}} S_{i}^{\prime}(\omega)
\end{gathered}
$$

where

$$
\begin{gathered}
V_{i j}=\frac{1}{c \rho l_{(l)} h_{1}}\left[-Q \frac{\partial\left\langle\alpha_{i}\right\rangle}{\partial T_{j}}-\frac{\partial\left\langle I_{i}\right\rangle}{\partial T_{j}}\right. \\
\left.+\frac{\partial\left\langle A_{i}\right\rangle}{\partial T_{j}}\right]_{T=\uparrow}
\end{gathered}
$$

For a stable equilibrium state $\hat{T}$ the feedback matrix $V$ is negative definite (i.e the real part of all eigenvalues of $V$ is negative).

Assuming that the characteristic time scale of the random forcing is small compared with the time scale of the climatic response, so that the spectrum of the atmospheric input can be regarded as white in the climatic frequency range, the variance cross spectrum $G_{i j}(\omega)$ of the surface temperature $T_{i}$ can be calculated from (2.7) as (cf. (I))

$G_{i j}(\omega)=H_{i k} H_{j l}^{*} F_{k l}(0)$

where $H=(i \omega I-V)^{-1}(I=$ unit matrix $)$ and $F_{k l}(0)$ is the cross spectrum of the random forcing at zero frequency

$$
F_{k l}(0)=\frac{1}{2 \pi\left(c \rho h_{1}\right)^{2} l_{(k)} l_{()}} \int_{-\infty}^{\infty}\left\langle S_{k}^{\prime}(t+\tau) S_{l}^{\prime}(t)\right\rangle d \tau
$$

\section{(b) Two layer ocean model}

For larger time scales, the "copper plate" model of the ocean in terms of a single homogeneous layer is clearly inappropriate. Although an accurate description will not be possible without including a full ocean circulation model, a more realistic representation of the thermal response of the ocean may be attempted by the following two layer model. The upper layer is again a completely mixed homogeneous layer of depth $h_{1}$. In the lower layer of depth $h_{2}(=4500 \mathrm{~m})$ a vertical heat flux occurs through turbulent heat conduction. The simplest models of the vertical thermal structure of the oceans are based on this concept (Munk, 1966). The vertical temperature profile in the lower layer is then determined by the (one dimensional) heat conduction equation

$$
\frac{\partial T}{\partial t}=\frac{\gamma}{\rho c} \cdot \frac{\partial^{2} T}{\partial z^{2}}
$$

with the boundary conditions

$$
T\left(z=a-h_{1}\right)=T(a) ;\left.\frac{\partial T}{\partial z}\right|_{a-h_{1}-h_{2}}=0 .
$$

The solution of (2.11) for a given Fourier component is given by

$$
\begin{aligned}
& T(z, \omega)=T(a, \omega) \\
& \left.\times \frac{\left.\exp \left[\frac{1+i}{a_{1}} z\right]+\exp \left[\frac{2(1+i)}{a_{1}}\left(a-h_{1}-h_{2}\right)\right]\right\}}{\exp \left[-\frac{1+i}{a_{1}} z\right]}\right\} \\
& \exp \left[\frac{1+i}{a_{1}}\left(a-h_{1}\right)\right]\left\{1+\exp \left[-\frac{2(1+i)}{a_{1}} h_{2}\right]\right\}
\end{aligned}
$$

where $a_{1}=\sqrt{2 \gamma / \omega \rho c}$.

The effective turbulent heat conductivity $\gamma$ of the ocean is taken to be $1 \mathrm{cal} \mathrm{cm}-1{ }^{\circ} \mathrm{C}^{-1} \mathrm{sec}^{-1}$ (Munk, 1966; Roether et al., 1970).

The boundary condition of zero heat flux through the ocean floor is not strictly correct, but this approximation is not critical. It can be shown that including the heat fluxes into the continents and the ocean floor reduces the power spectrum of $T$ for frequencies in the range of $10^{-1} \mathrm{cpy}$ to $10^{-5}$ cpy by only 3 to $5 \%$. Outside this range the correction is still smaller. The effect is weak because of the relatively small heat conductivity of the earth's crust $\left(0.0023 \mathrm{cal} \mathrm{cm}^{-1}{ }^{\circ} \mathrm{C}^{-1} \mathrm{sec}^{-1}\right)$ as compared with the oceans $\left(1 \mathrm{cal} \mathrm{cm}^{-1}{ }^{\circ} \mathrm{C}^{-1} \mathrm{sec}^{-1}\right)$.

The differential equation $(2.4)$ for the surface temperature takes for the two layer model the form

$$
\begin{aligned}
& l_{(i)} c \rho\left[h_{1} \frac{\partial T_{i}(a, t)}{\partial t}+\frac{\partial}{\partial t} \int_{a-h_{1}-h_{2}}^{a-h_{1}} T_{i}(z, t) d z\right] \\
& \quad=\left\langle R_{i}\right\rangle+\left\langle A_{i}\right\rangle+S_{i}^{\prime}
\end{aligned}
$$

Tellus 29 (1977), 5 
Substituting (2.12), one can easily carry out the integration over the temperature profile on the lefthand side of (2.13), and one obtains

$$
\begin{gathered}
i \omega g(\omega) T_{i}(a, \omega)=V_{i j}\left[T_{j}(a, \omega)-\hat{T}_{j}(a, \omega)\right] \\
+\frac{1}{\operatorname{col}_{(i)} h_{1}} S_{i}^{\prime}
\end{gathered}
$$

Equation (2.14) differs from eq. (2.7) for the copper plate model only through the additional complex heat capacity factor

$$
\begin{aligned}
g(\omega)=1+ & \frac{a_{1}}{2 h_{1}} \cdot \frac{\sinh \beta+\sin \beta}{\cosh \beta+\cos \beta} \\
& -i \frac{a_{1} \sinh \beta-\sin \beta}{2 h_{1} \cosh \beta+\cos \beta}
\end{aligned}
$$

where

$\beta=\frac{2 h_{2}}{a_{1}}$.

For small $\omega$ the imaginary part of $g(\omega)$ vanishes and the real part approaches the constant value $g(\omega \rightarrow 0)=1+h_{2} / h_{1}$. This is because long-period temperature waves affect deep layers of the ocean, and as $\omega \rightarrow 0$ the thermal capacity is given simply by $c \rho\left(h_{1}+h_{2}\right)$. For large $\omega$ the real part of $g(\omega)$ approaches 1 , and the imaginary part vanishes as $1 / \sqrt{\omega}$. The imaginary part of $g(\omega)$ corresponds formally to a term $\sqrt{2 \gamma \omega / p c} / 2 h_{1}$ contributing negatively to the diagonal elements of the feedback matrix $V_{i j}$. However, this negative feedback is of little significance for the spectral response, since for large $\omega$ the feedback becomes small generally compared with the thermal inertia term.

As an alternative description of the lower ocean layer an upwelling term can be included. This is perhaps more consistent with the one-dimensional heat balance model of the deep ocean on which estimates of the turbulent heat diffusion coefficient are normally based. The vertical temperature profile is then determined by the heat balance equation

$\frac{\partial T}{\partial t}=\frac{\gamma}{\rho c} \cdot \frac{\partial^{2} T}{\partial z^{2}}-w \cdot \frac{\partial T}{\partial z}$

where the upwelling velocity $w=1.2 \mathrm{~cm} /$ day (Munk, 1966). The analysis is the same as above except, that the complex heat capacity factor $g(\omega)$ is modified.

\section{(c) Parameterization}

In order to calculate the power spectrum of $T$ according to (2.9) we have to prescribe the temperature dependence of the albedo, the infrared emission and the meridional flux term (cf. (2.8)). We take the parameterization of the infrared emission $\langle I(T)\rangle$ and the average energy gain due to the meridional flux $\langle A(T)\rangle$ as functions of the surface temperature from Budyko (1969)

$\langle I(T)\rangle=A_{1}+B_{1} T$

$\langle A(T)\rangle=\gamma_{1}(\bar{T}-T)$

where $A_{1}, B_{1}$ and $\gamma_{1}$ are empirical constants, and $\bar{T}$ is the global average of the surface temperature, $\bar{T}(t)=\int_{0}^{\pi / 2} T(\theta, t) \cos \theta d \theta$

We shall consider two parameterizations of the temperature dependence of the albedo, Sellers' (1969) relation

$\alpha(T)= \begin{cases}a(\theta)-b T & T<283 \mathrm{~K} \\ a(\theta)-b \cdot 283 & T>283 \mathrm{~K}\end{cases}$

and a second relation which assumes that there is no albedo feedback north of the yearly averaged ice-boundary. In the second case the albedo feedback takes place only in the region between the snowfall margin and the ice-boundary. Assuming a cosine-form for the annual temperature cycle

$T(t)=T_{m}-a_{3} \cos 2 \pi t$

the yearly averaged albedo as a function of temperature is given by

$\alpha\left(T_{m}\right)= \begin{cases}\alpha_{E} & T_{m}>a_{3} \\ \alpha_{E}+\left(\alpha_{s}-\alpha_{E}\right) \frac{1}{\pi} \arccos \left(T_{m} / a_{3}\right) & \left|T_{m}\right|<a_{3} \\ \alpha_{s} & T_{m}<-a_{3}\end{cases}$

where $\alpha_{E}(\theta)$ is the snow-free albedo at latitude $\theta, \alpha_{s}$ is the albedo in presence of snowcover, $T_{m}$ is the yearly averaged surface air temperature, and $\pm a_{3}$, taken to be $\pm 10^{\circ} \mathrm{C}$, designates the yearly averaged temperature at the snowfall margin and the iceboundary, respectively.

\section{(d) Stochastic forcing}

The power spectrum of the random forcing (2.10) has to be estimated from real data. Unfor- 
tunately, time series data of the relevant energy flux term entering in the Budyko-Sellers model, or of the zonally averaged humidity and cloudiness, are not readily available. However, order of magnitude estimates can be obtained by scaling considerations.

Three rapidly fluctuating weather variables appear in the right-hand side of (2.4).

$S_{i}^{\prime}=A_{i}^{\prime}+b_{1} n_{i}^{\prime}+b_{2} q_{i}^{\prime}$

The first term is the variation $A_{i}^{\prime}$ of the meridional flux term. The second and third terms arise from the variations $n_{i}^{\prime}, q_{i}^{\prime}$ in cloudiness and humidity, respectively. The constants $b_{1}$ and $b_{2}$ can be derived from the parameterizations of the albedo and infrared emission in terms of these variables. However, the estimates suggest that the contributions of $n^{\prime}$ and $q^{\prime}$ to the random forcing are small compared with the meridional flux term, and for simplicity we shall therefore retain only the term $A_{i}^{\prime}$.

Equation (2.10) is thus given by

$$
\begin{aligned}
F_{i j}(0)= & \frac{1}{2 \pi\left(c \rho h_{1}\right)^{2} l_{(i)} l_{(j)}} \int R_{i j}^{A}(\tau) d \tau=\frac{1}{2 \pi c \rho l_{(i)} h_{1}} \\
& \times R_{i j}^{A}(0) \bar{\tau}
\end{aligned}
$$

where $R_{i j}^{A}$ is the covariance function of $A$ and $\bar{\tau}$ (by definition through the second form of the equation) is the integral correlation time scale of $A$.

To estimate $F_{i j}(0)$, the integral correlation time $\bar{\tau}$ was taken as 5 days. It was assumed further that the diagonal elements $\sigma_{A}^{2}=R_{i i}^{A}(0)$ were constant for all latitudes $i$, and that the r.m.s. deviation $\sigma_{A}$ was equal to the mean of $A$ at $55^{\circ}$ latitude. This value is not inconsistent with the analysis of Oort (in preparation), which shows that the r.m.s. deviations of the sensible and latent heat flux at various heights in the atmosphere generally lie in the range of $50 \%$ to $100 \%$ of the mean.

The horizontal correlation length was assumed to be of order $1500 \mathrm{~km}$ ( $15^{\circ}$ latitude); specifically with a spacing of $5^{\circ}$ the cross-correlation matrix $R_{i j}^{A}$ was set equal to

$$
\begin{aligned}
& R_{i, i \pm 1}^{A}(0)=\frac{3}{4} R_{i i}^{A}(0) \\
& R_{i, i \pm 2}^{A}(0)=\frac{1}{2} R_{i i}^{A}(0) \\
& R_{i, i \pm 3}^{A}(0)=\frac{1}{4} R_{i i}^{A}(0) \\
& R_{i, i \pm n}^{A}(0)=0 \text { for } n>3
\end{aligned}
$$

\section{Results}

Equation (2.9) was evaluated numerically. Fig. 1 shows the temperature spectrum at different latitudes for a Sellers-type albedo feedback and a one-layer ocean model, with a mixed layer depth

$h_{1}=\left\{\begin{array}{rr}10 \mathrm{~m} & \theta>72^{\circ} \\ 50 \mathrm{~m} & 30^{\circ}<\theta<72^{\circ} \\ 25 \mathrm{~m} & \theta<30^{\circ}\end{array}\right.$

These values reflect the fact that mixed layer depth is larger at higher latitudes than at lower latitudes, because of the stronger wind-mixing, and that the ocean is partly covered with ice for $\theta>72^{\circ}$. The wind-mixing in polar regions is less efficient, so that a smaller mixed layer is generated.

The spectra increase with decreasing frequency flattening at lower frequencies because of the net negative feedback, as described in $I$. The undulations around $f \approx 10^{-1}$ cpy are caused by the coupling between latitudes through the meridional flux term, which results in a superposition of different relaxation rates from different latitudes. (The matrix of eigensolutions of the linear system is not diagonal with respect to the latitude index $i$.) For large frequencies the level of the curve is mainly determined by the heat capacity of the system. Thus the spectrum at the pole is higher than at $55^{\circ}$ latitude because of the smaller thermal inertia of the mixed layer ( $10 \mathrm{~m}$ depth) at high latitudes.

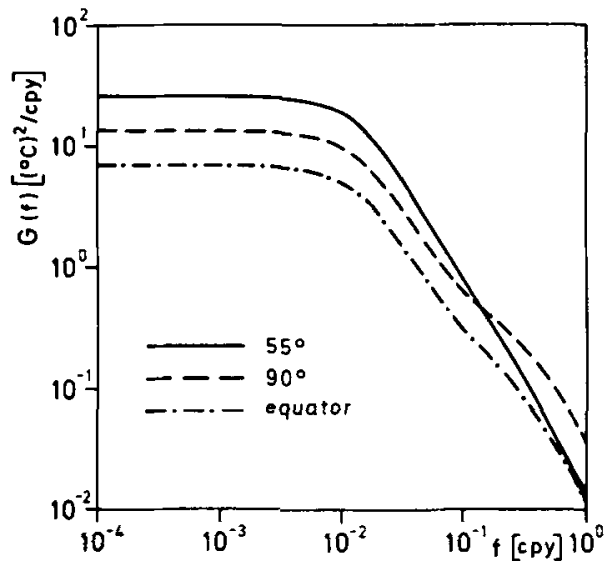

Fig. 1. Theoretical variance spectra of the surface temperature at different latitudes. (Sellers'albedo; $b=0.009$; single-layer ocean model.) 


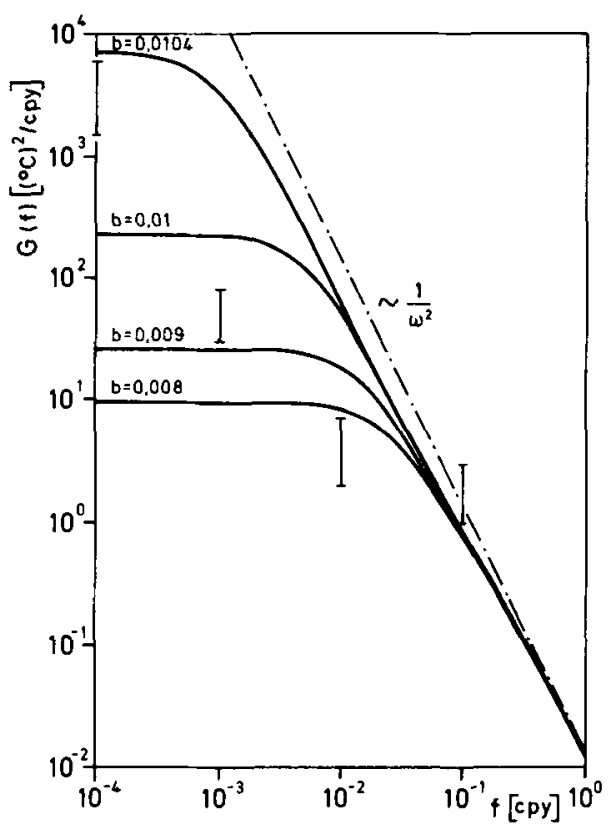

Fig. 2. Theoretical variance spectra of the surface temperature at $55^{\circ}$ latitude for different albedo feedback parameters $b$. (Sellers' albedo; single-layer ocean model.) The bars indicate temperature data for Central England, from Kutzbach \& Bryson (1974).

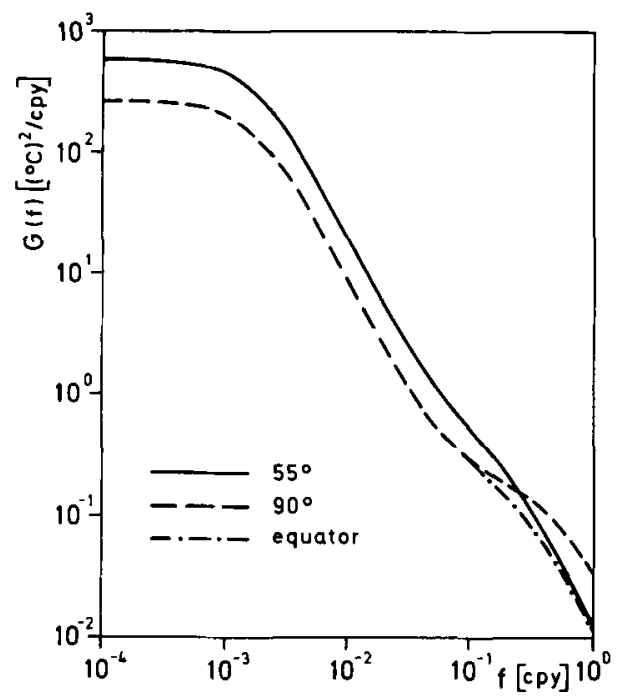

Fig. 3. Theoretical variance spectra of the surface temperature at different latitudes. (Arccos-albedo; $b_{1}=$ 0.006 ; single-layer ocean model.)

The spectral levels at zero frequency are determined mainly by the net negative feedback. For diagonal $V\left(V_{i j}=\lambda_{(j)} \delta_{i j}\right)$ the zero frequency level is given by $F_{i l}(0) / \lambda_{i}^{2}$. Because of the large positive albedo feedback the level is higher at latitude $55^{\circ}$ than at the pole, where the albedo feedback is decreased due to the smaller solar radiation $Q$. For the ice- and snow-free low latitudes there is again no positive albedo feedback, and the low zerofrequency level is governed solely by the negative feedback from the infrared emission and the meridional flux.

In Fig. 2 the power spectrum of the surface temperature at latitude $55^{\circ}$ is plotted for different albedo feedback parameters $b$. The straight line is the spectrum without feedback ( $\omega^{-2}$ spectrum). The bars indicate the range of experimental and historical data for Central England, from Kutzbach \& Bryson (1974). (Note, however, that the variance for an individual temperature record will be higher than the variance of zonally averaged temperatures considered here.)

Increasing $b$ corresponds to an increase of the positive feedback and thus a decrease of the resultant negative feedback. The feedback dependence is most pronounced at low frequencies, as expected. For $b>0.0105$ the resultant feedback becomes positive and the system becomes unstable.

The strong dependence of the spectra on the feedback parameter illustrates in another way the well known sensitivity of the Budyko-Sellers model to small changes in the model parameters. This is due to the fact that the model has been tuned to lie very close to the instability point.

Figs. 3 and 4 correspond to Figs. 1 and 2 except that Sellers' albedo (2.17) has been replaced by the arccos-albedo (2.19). At large frequencies the spectra in both cases are nearly identical, because the heat capacities of the system are the same. The differences occur at small frequencies, where the albedo feedback has a stronger influence. Because the arccos-albedo yields zero albedo feedback beyond the snow and ice limits $\left(72^{\circ}\right.$ and $42^{\circ}$ latitude), and the random forcing is assumed latitude independent, the spectra at the equator and at the pole are identical for small frequencies. Albedo feedback parameters $b_{1}=\left(\alpha_{E}-\alpha_{9}\right) / a_{3} \pi$ larger than 0.00607 lead again to an unstable growth of the temperature variations (Fig. 4).

Fig. 5 shows the effect of including a second ocean layer. At large frequencies the spectrum is determined mainly by the mixed layer and decays as $\omega^{-2}$, as before. As $f$ decreases, deeper layers of the ocean are affected by the temperature oscillations at the surface, so that the thermal inertia 


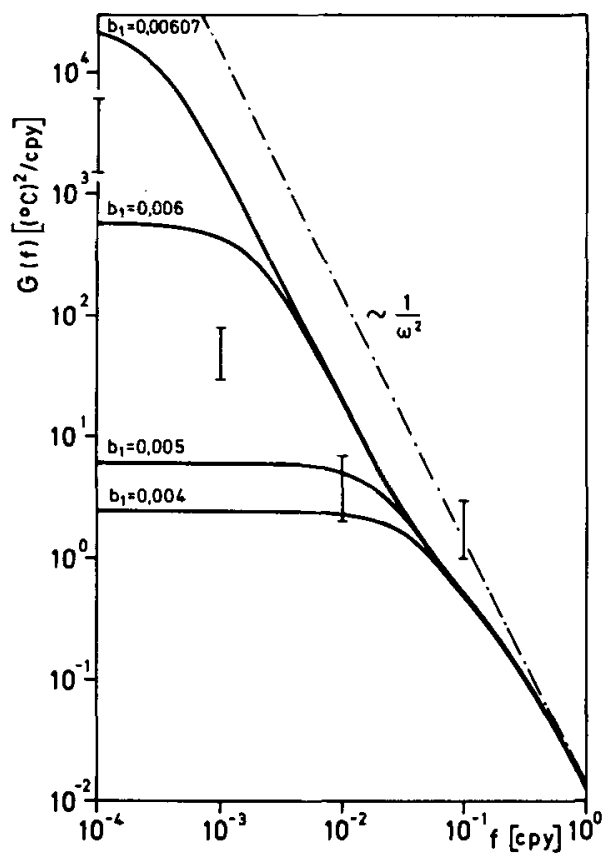

Fig. 4. Theoretical variance spectra of the surface temperature at $55^{\circ}$ latitude for different albedo feedback parameters $b_{1}$. (Arccos-albedo; single-layer ocean model.) The bars indicate temperature data for Central England, from Kutzbach \& Bryson (1974).

increases and the spectrum is reduced. This effect continues until the temperature waves reach the bottom of the ocean. Then the thermal inertia becomes constant, and the spectral response returns again to a $\omega^{-2}$ law at a lower level corresponding to the larger heat capacity of the entire ocean column. At lower frequencies, finally, the spectrum flattens again because of the negative feedback. The level at zero frequency is the same as for the one-layer model, since for very low frequencies all thermal inertia effects become unimportant; the level at $\omega=$ 0 can be changed only by changing the feedback $V_{u}$ or random forcing $F_{u}(0)$.

Upwelling has the effect of reducing the depth of penetration of the temperature waves into the lower layer.

Thus the characteristic low-frequency response times of the system are reduced by almost an order of magnitude and the variance spectrum in the range $10^{-5}<f<10^{-3}$ is accordingly higher.

The total variance of the temperature spectrum is $1.2\left({ }^{\circ} \mathrm{C}\right)^{2}$ (for the two-layer ocean model, Fig. 5).

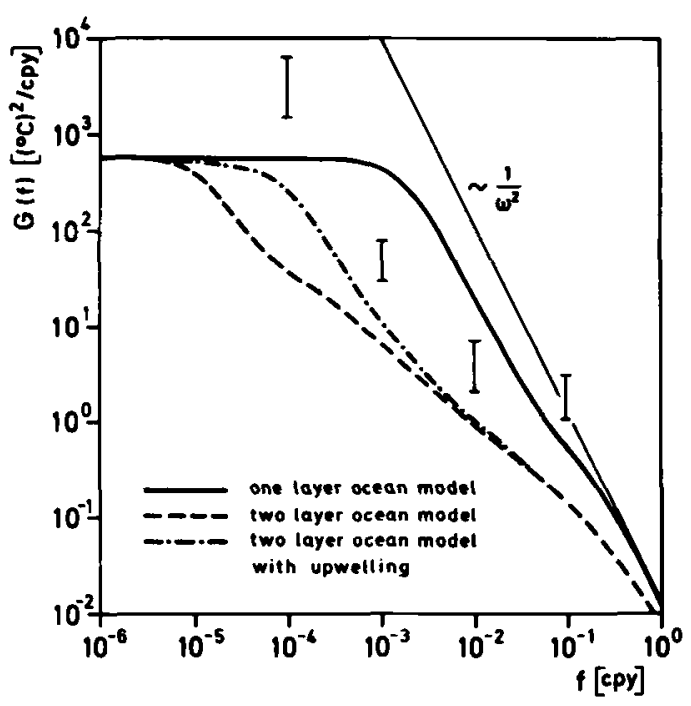

Fig. 5. Influence of a second ocean layer on the variance spectrum of the surface temperature at $55^{\circ}$ latitude. (Arccos type albedo feedback, $b_{1}=0.006$.) The bars indicate temperature data for Central England, from Kutzbach \& Bryson (1974).

This value may be compared with changes produced by variations in the solar radiation. Using essentially the same model, Budyko (1969) found that a $1 \%$ decrease in solar radiation caused a change of the mean temperature of the earth's surface by $1.5^{\circ} \mathrm{C}$. A similar result was obtained by Manabe \& Wetherald (1967) using a high resolution General Circulation Model: they found a $1.2^{\circ} \mathrm{C}$ change in the mean temperature of the earth's surface for a $1 \%$ change in the solar radiation.

Thus the internal stochastic forcing of the climate system by short time-scale weather variability generates a temperature variance of the same order as produced by $1 \%$ variations in the solar radiation.

It should be noted, however, that the temperature variations were computed here for small excursions about an equilibrium state, assuming linear feedback and a random forcing independent of the climate state. For larger changes (ice ages) the dependence of the feedback coefficients and random forcing on the climate state will need to be taken into account in the framework of a more general theory based on the Fokker-Planck equation (cf. I). 


\section{Conclusions}

The general features of the observed spectra of the surface temperature variability in the range of climatic time scales from 10 to $10^{4}$ years can be explained by a stochastic forcing model, in which the temperature fluctuations are generated through internal random forcing.

Both the qualitative form of the spectrum and the order of magnitude of the energy levels are consistent with observations-more can clearly not be expected from the highly idealized Budyko-Sellers model used. Different positive albedo feedbacks affect the shape and the level of the spectra very strongly at small frequencies, while the heat capacity is dominant for large frequencies.

There exists a critical value of the albedo feedback factor beyond which the spectral response becomes infinite (net positive feedback). This instability value is the same as that found by Held \& Suarez (1974), and is not far removed from the mean operating value normally assumed for the Budyko-Sellers model. The introduction of a second ocean layer reduces the level of the spectra for frequencies greater than $10^{-5}$ years $^{-1}$. The level at zero frequency is not changed. The results demonstrate the importance of constructing more realistic ocean-cryosphere models for studying lower frequency climate phenomena.

\section{Acknowledgement}

I wish to thank K. Hasselmann for many helpful discussions.

\section{REFERENCES}

Adem, J. 1965. Experiments Aiming at Monthly and Seasonal Numerical Weather prediction. Mon. Weather Rev. 93, 495-503.

Budyko, M. I. 1969. The effect of solar radiation variations on the climate of the earth, Tellus $21,611-$ 619.

Frankignoul, C. \& Hasselmann, K. 1977. Stochastic climate models, Part 2, Application to sea-surface temperature anomalies and thermocline variability. Tellus 29, 289-305.

Hasselmann, K. 1976. Stochastic climate models, Part 1, Theory. Tellus 28, 473-485.

Held, I. M. \& Suarez, M. J. 1974. Simple albedo feedback models of the icecaps. Tellus 26, 613-629.

Kutzbach, J. E. \& Bryson, R. A. 1974. Variance Spectrum of Holocene Climatic Fluctuations in the North Atlantic Sector. J. of the Atmos. Sci. 31, 19581963.

Manabe, S. \& Wetherald, R. 1967. Thermal equilibrium of the atmosphere with a given distribution of relative humidity. J. of the Atmos. Sci. 24, 241-259.

Munk, W. H. 1966. Abyssal recipes. Deep-Sea Res. 13, 707-730.

Oort, A. H. 1976. The Interannual Variability of Atmospheric Statistics, NOAA Report (in preparation).

Roether, W., Münnich, K.-O. and Östlund, H. G. 1970. Tritium Profile at the North Pacific (1969) Geosecs Intercalibration Station. J. of Geophys. Res. 75, 76727675.

Schneider, S. H. \& Gal-Chen, Tzvi 1973. Numerical Experiments in Climate Stability. J. of Geophys. Res. 78, 6182-6194.

Sellers, W. D. 1969. A global climate model based on the energy balance of the earth-atmosphere system. $J$. of Appl. Meteorol. 8, 392-400.

Sellers, W. D. 1973. A new Global Climatic Model. J. of Appl. Meteorol. 12, 241-254.

\section{СТОХАСТИЧЕСКИЕ МОДЕЛИ КЛИМАТА. ЧАСТЬ 3.}

\section{ПРИМЕНЕНИЕ К ЗОНАЛЬНО ОСРЕДНЕННЫМ ЭНЕРГЕТИЧЕСКИМ МОДЕЛЯМ}

Рассматривается стохастическая модель БудыкоСеллерса в которой в отличие от обычных статистико-динамических моделей климата неосредненные погодные флуктуации сохранены в качестве случайного внутреннего вынуждающего источника. Поэтому климатические переменные более не являются детерминистическими, а ока- зываются случайными переменными, которые могут быть характеризованы энергетическим спектром. Вычисленные спектры зонально и годично осредненной температуры поверхности согласуются с наблюдениями как в качественной структуре спектров, так и в порядках величн уровней энергин. 